\title{
NEON AND SULFUR ABUNDANCES OF PLANETARY NEBULAE IN THE MAGELLANIC CLOUDS
}

\author{
J. Bernard-Salas, ${ }^{1}$ S. R. Pottasch, ${ }^{2}$ S. Gutenkunst, ${ }^{1}$ P. W. Morris, ${ }^{3}$ and J. R. Houck ${ }^{1}$ \\ Received 2007 January 31; accepted 2007 September 9
}

\begin{abstract}
The chemical abundances of neon and sulfur for 25 planetary nebulae (PNe) in the Magellanic Clouds are presented. These abundances have been derived using mainly infrared data from the Spitzer Space Telescope. The implications for the chemical evolution of these elements are discussed. A comparison with similarly obtained abundances of Galactic PNe and $\mathrm{H}$ II regions and Magellanic Cloud $\mathrm{H}$ in regions is also given. The average neon abundances are $6.0 \times 10^{-5}$ and $2.7 \times 10^{-5}$ for the PNe in the Large and Small Magellanic Clouds, respectively. These are $\sim 1 / 3$ and $1 / 6$ of the average abundances of Galactic planetary nebulae to which we compare. The average sulfur abundances for the $\mathrm{LMC}$ and SMC are, respectively, $2.7 \times 10^{-6}$ and $1.0 \times 10^{-6}$. The $\mathrm{Ne} / \mathrm{S}$ ratio $(23.5)$ is on average higher than the ratio found in Galactic PNe (16), but the range of values in both data sets is similar for most of the objects. The neon abundances found in PNe and $\mathrm{H}$ II regions agree with each other. It is possible that a few (3-4) of the PNe in the sample have experienced some neon enrichment, but for two of these objects the high $\mathrm{Ne} / \mathrm{S}$ ratio can be explained by their very low sulfur abundances. The neon and sulfur abundances derived in this paper are also compared to previously published abundances using optical data and photoionization models.
\end{abstract}

Subject headings: infrared: general — ISM: abundances — Magellanic Clouds — planetary nebulae: general

Online material: color figure

\section{INTRODUCTION}

Stars of low and intermediate mass $\left(\sim 1-8 M_{\odot}\right)$ become planetary nebulae after they evolve off the asymptotic giant branch (AGB; Iben \& Renzini 1983). In the planetary nebula (PN) phase the hot central star ionizes the previously ejected material, which then emits copious amounts of emission lines of different ions. These emission lines are ideal for studying the chemical composition of the gas. The abundances of elements such as carbon, nitrogen, and oxygen can be used to give information on the nucleosynthesis history of the progenitor star. Other elements, such as neon, sulfur, and argon are not supposed to be altered in the course of evolution of low- and intermediate-mass stars and are therefore indicators of the chemical composition at the epoch of formation (Marigo et al. 2003).

It is for these reasons that PNe have been the subject of many spectroscopic studies over the years. Due to limitations in the observations, the bulk of this spectroscopic work has been focused on analyzing PNe in the Milky Way. However, observations of PNe outside the Galaxy are very important because one can probe different metallicity regions and, unlike Galactic PNe, the distance is known, which allows one to relate the abundance to the central star luminosity. During the last few years several papers (e.g., Magrini et al. 2001, 2003; Corradi et al. 2005 and references therein) have been devoted to identifying PNe outside the Galaxy. As a consequence, the number of PNe known in the Local Group keeps increasing. The Large and Small Magellanic Clouds (hereafter LMC and SMC, respectively) are ideal candidates from which to obtain spectroscopic observations of $\mathrm{PNe}$.

Aller et al. (1981) and Aller (1983) published optical spectroscopic data for six PNe in the LMC and seven in the SMC, respectively. In a follow-up paper Aller et al. (1987) presented

\footnotetext{
1 Center for Radiophysics and Space Research, Cornell University, 222 Space Sciences Building, Ithaca, NY 14853-6801.

2 Kapteyn Astronomical Institute, 9700 AV, Groningen, Netherlands.

3 NASA Herschel Science Center, IPAC/Caltech, MS 100-22, Pasadena, CA 91125 .
}

ultraviolet data from the International Ultraviolet Explorer (IUE) satellite of $12 \mathrm{PNe}$ in the Magellanic Clouds (MCs) and derived abundances for several elements. Ultraviolet data are essential in order to derive abundances of elements such as carbon and nitrogen. Optical spectroscopy and abundances of $71 \mathrm{MC}$ PNe were presented by Monk et al. (1988). In the early 1990s Meatheringham \& Dopita (1991a, 1991b) obtained optical spectroscopy of over 100 PNe in the MCs. Morgan \& Parker (1998) presented FLAIR spectroscopy of $97 \mathrm{PNe}$ in the LMC, which included fluxes of the [O III], [S II], [N II], and He II lines. Using optical and IUE data, Peña et al. (1997) studied a sample of MC PNe with Wolf-Rayet (WR) nuclei and found that the distribution of spectral type was different from those of Galactic WR PNe. Stanghellini et al. (2002, 2003) have characterized optically a large number of $\mathrm{PNe}$ in the MCs using Hubble Space Telescope (HST) observations. More recently, Leisy \& Dennefeld (2006) have derived the abundances of several elements for a large sample of PNe in the MCs using optical data.

Despite their importance, infrared spectroscopic studies of $\mathrm{PNe}$ in the MCs are scarce in the literature. This is mainly because full integrated spectra in the infrared can only be achieved from space. IRAS detected several PNe in the MCs (Zijlstra et al. 1994), mainly at 12 and $25 \mu \mathrm{m}$, but some of the identifications are dubious. The SWS spectrograph (de Graauw et al. 1996) on board Infrared Space Observatory (ISO) did an excellent job studying nearby $\mathrm{PNe}$, but it did not have enough sensitivity to allow the study of PNe outside the Galaxy. The Spitzer Space Telescope (Werner et al. 2004) with its increased sensitivity enables us to observe PNe outside the Milky Way (Bernard-Salas et al. 2006, 2004). The importance of using infrared lines when deriving abundances has been highlighted by Rubin et al. (1988), and we summarize these reasons here. Infrared lines are little affected by extinction, as opposed to optical or UV lines. Uncertainties in the electron temperature or fluctuations in the temperature within the nebula are not important when using infrared lines because they originate from levels very close to the ground level. In addition, many ions emit in the infrared, and therefore the use of ionization correction 
factors (ICFs) can be greatly reduced by including infrared observations. This is especially true for neon, sulfur, and argon. Finally, while it is not discussed in this paper, emission of dust can be studied in this part of the electromagnetic spectrum.

In this paper we present Spitzer high-resolution spectroscopic observations of $25 \mathrm{PNe}$ in the MCs (18 in the LMC and seven in the SMC). This paper focuses on the emission of fine-structure lines and their use in the abundance determination. The measured lines are used to derive abundances for sulfur and neon. These abundances are mainly compared to Galactic PNe abundances from Pottasch \& Bernard-Salas (2006), as well as Galactic, MC, M33, and M83 $\mathrm{H}$ II region abundances from Martín-Hernández et al. (2002), Vermeij \& van der Hulst (2002), and Rubin et al. (2007). All of these abundances have been derived using infrared data and in a similar way to that presented in this paper. Dust features present in the spectra, such as polycyclic aromatic hydrocarbons (PAHs) and silicates, will be discussed in another paper (J. Bernard-Salas et al., in preparation) which makes use of the Spitzer low-resolution spectra from 5 to $37 \mu \mathrm{m}$.

\section{OBSERVATIONS AND DATA REDUCTION}

The observations were made using the Infrared Spectrograph (IRS; Houck et al. 2004) on board the Spitzer Space Telescope and resulted in high- and low-resolution spectra of $25 \mathrm{PNe}$. These observations were part of the GTO program (ID 103) and were taken between 2005 March and November. The object name and AOR key (Astronomical Observation Request key) numbers for each observation are given in the first and second columns of Table 1. The nomenclature given by Sanduleak et al. (1978) is adopted throughout the paper. In addition, the analysis includes data on SMP LMC 31, and abundances on SMP LMC 83 derived by Bernard-Salas et al. (2004). These data were taken during in orbit check-out (IOC). There are two high-resolution modules in the IRS, named Short-High and Long-High (SH and LH, respectively). Together, they cover the wavelength region between 10 and $37 \mu \mathrm{m}$ at a resolution of 600 . The reader should refer to the paper by Houck et al. (2004) for more information on the IRS instrument. We used coordinates given by Stanghellini et al. (2002, 2003) and Leisy et al. (1997) and performed blue peak-up acquisition on a nearby star to obtain accurate pointing $\left(0.4^{\prime \prime}\right)$. Figure 1 shows the position of the LMC PNe on an IRAC image from the SAGE team (Meixner et al. 2006).

The data were processed through a copy of the S13.2 version of the Spitzer Science Center's pipeline, which is maintained at Cornell, and using a script version of Smart (Higdon et al. 2004). The reduction started from the droop images. These are equivalent to the most commonly used bcd data and only lack the flatfield and stray-cross-light removal (which is only important for bright sources). Rogue pixels which are especially notorious in the LH module were removed using the irsclean ${ }^{4}$ tool. The rogue pixels were first flagged using a campaign mask and then removed. If different cycles (repetitions) were present for a given observation these were combined using the mean to improve the $\mathrm{S} / \mathrm{N}$. The two-dimensional images were extracted using fullaperture extraction. The calibration was performed by dividing the resultant spectrum by that of the calibration star $\xi$ Dra (extracted in the same way as the target) and multiplying by its template (Cohen et al. 2003; G. C. Sloan et al., in preparation). Finally, glitches which were not present in both nod positions or in the overlapping region between orders were removed manually.

There is an expected mismatch between the SH and LH spectra. This mismatch is due to differences in the background contri-

\footnotetext{
${ }^{4}$ This tool is available from the SSC Web site: http://ssc.spitzer.caltech.edu.
}

TABLE 1

Adopted Parameters for Abundance Determination

\begin{tabular}{|c|c|c|c|c|c|}
\hline Object & AOR key & $\log \left(F_{\mathrm{H} \beta}\right)^{\mathrm{a}, \mathrm{b}}$ & $C_{\mathrm{H} \beta}{ }^{a}$ & $\begin{array}{c}T_{e}^{\mathrm{a}} \\
(\mathrm{K})\end{array}$ & $\begin{array}{c}N_{e}^{\mathrm{a}} \\
\left(\mathrm{cm}^{-3}\right)\end{array}$ \\
\hline SMP LMC $02 \ldots \ldots \ldots$ & 4946944 & -13.18 & 0.06 & 11600 & $30020^{\mathrm{c}}$ \\
\hline SMP LMC $08 \ldots \ldots \ldots$ & 15902464 & -13.74 & 0.23 & 11000 & 5500 \\
\hline SMP LMC $11 \ldots \ldots \ldots$ & 4947712 & -13.94 & 0.31 & 25000 & 6200 \\
\hline SMP LMC $13 \ldots \ldots \ldots$ & 4947968 & -12.88 & 0.09 & 13600 & 3800 \\
\hline SMP LMC $28 \ldots \ldots \ldots$ & 4948224 & -13.57 & 0.32 & 10000 & 2000 \\
\hline SMP LMC $31 \ldots \ldots \ldots$ & 7459584 & -12.92 & 0.54 & 12800 & 6800 \\
\hline SMP LMC $35 \ldots \ldots . .$. & 4948736 & -12.81 & 0.04 & 13300 & 1600 \\
\hline SMP LMC $36 \ldots \ldots . .$. & 4949248 & -12.72 & 0.41 & 15000 & $3000^{\mathrm{c}}$ \\
\hline SMP LMC $38 \ldots \ldots \ldots$. & 12633600 & -12.62 & 0.21 & 13000 & 9800 \\
\hline SMP LMC $40 \ldots \ldots \ldots$. & 4949504 & -13.25 & 0.20 & 13900 & 1100 \\
\hline SMP LMC $53 \ldots . . . .$. & 15902720 & -12.62 & 0.13 & 13700 & 4000 \\
\hline SMP LMC $58 \ldots \ldots \ldots$. & 4950784 & -12.54 & 0.11 & 12100 & 20000 \\
\hline SMP LMC $61 \ldots \ldots \ldots$ & 12633856 & -12.48 & 0.22 & 10800 & 26000 \\
\hline SMP LMC $62 \ldots \ldots \ldots$ & 4951040 & -12.30 & 0.07 & 15800 & 4400 \\
\hline SMP LMC $76 \ldots \ldots \ldots$ & 4951296 & -12.54 & 0.34 & 11600 & 13600 \\
\hline SMP LMC $78 \ldots \ldots \ldots$. & 15902208 & -12.60 & 0.21 & 14200 & 4300 \\
\hline SMP LMC $85 \ldots \ldots \ldots$ & 4952320 & -12.42 & 0.26 & 10500 & 31400 \\
\hline SMP LMC $87 \ldots \ldots . .$. & 4952576 & -12.91 & 0.25 & 19200 & 1900 \\
\hline SMP SMC $01 \ldots \ldots .$. & 4953088 & -12.85 & 0.287 & 11000 & 9600 \\
\hline SMP SMC $03 \ldots \ldots . .$. & 4953600 & -13.13 & 0.000 & 13800 & 5600 \\
\hline SMP SMC $06 \ldots \ldots \ldots$ & 4954112 & -12.80 & 0.385 & 15300 & 14700 \\
\hline SMP SMC $11 \ldots \ldots \ldots$. & 15902976 & -12.87 & 0.82 & 17600 & 1100 \\
\hline SMP SMC $22 \ldots \ldots \ldots$ & 4954624 & -12.94 & 0.165 & 18800 & 2500 \\
\hline SMP SMC $24 \ldots \ldots \ldots$ & 15901952 & -12.66 & 0.047 & 12700 & 1300 \\
\hline SMP SMC $28 \ldots \ldots \ldots$ & 4955136 & -13.18 & 0.200 & 20300 & 8800 \\
\hline
\end{tabular}

${ }^{a}$ Values taken from the following references (see $\S 3.2$ ): Leisy \& Dennefeld (2006); Meatheringham et al. (1998); Meatheringham \& Dopita (1991a, 1991b); Shaw et al. (2006); Stanghellini et al. (2002, 2005); Villaver et al. (2003, 2004); Wood et al. (1987).

${ }^{b}$ Flux in units of erg s ${ }^{-1} \mathrm{~cm}^{-2}$.

c Assumed electron density.

bution that falls into the slits because the slit size of the LH module is about 4.6 times larger in area than the SH slit. We do not scale the spectra because we are interested in the line fluxes and the nebulae are contained in both slits. These PNe are not extended at such distances. Diameters of PNe in the LMC given by Shaw et al. (2001) and Stanghellini et al. (1999) are usually less than $1^{\prime \prime}$, and only in a very few cases does the diameter reach $3^{\prime \prime}$ (still smaller than the SH slit width of $4.5^{\prime \prime}$ ). Representative examples of the full extracted high-resolution spectra from 10 to $37 \mu \mathrm{m}$ are shown in Figure 2. The spectrum of each object containing the low-resolution data $(5-37 \mu \mathrm{m})$ will be shown in J. Bernard-Salas et al. (in preparation).

\section{ANALYSIS}

\subsection{Line Emission}

Figure 3 shows an inset of the most relevant lines for the abundance determination present in the spectra of all the objects. The sample ranges from $\mathrm{PNe}$ showing high-excitation to lowexcitation lines (e.g., SMP LMC 85, SMP LMC 62). Most of the spectra show features (PAHs) characteristics of carbon-rich material (e.g., SMP LMC 36, SMP SMC 11 in Fig. 2), except for SMP LMC 53 and SMP LMC 62 (Fig. 2), which show amorphous silicates in emission at 9 and $18 \mu \mathrm{m}$, which is usually an indicator of an oxygen-rich environment (J. Bernard-Salas et al., in preparation).

The line fluxes are listed in Table 2. In addition to the lines listed in this table, other lines such as [Mg v] at $13.52 \mu \mathrm{m}$ have been measured for the PNe with higher $\mathrm{S} / \mathrm{N}$. The $[\mathrm{S}$ III] $33.48 \mu \mathrm{m}$ 


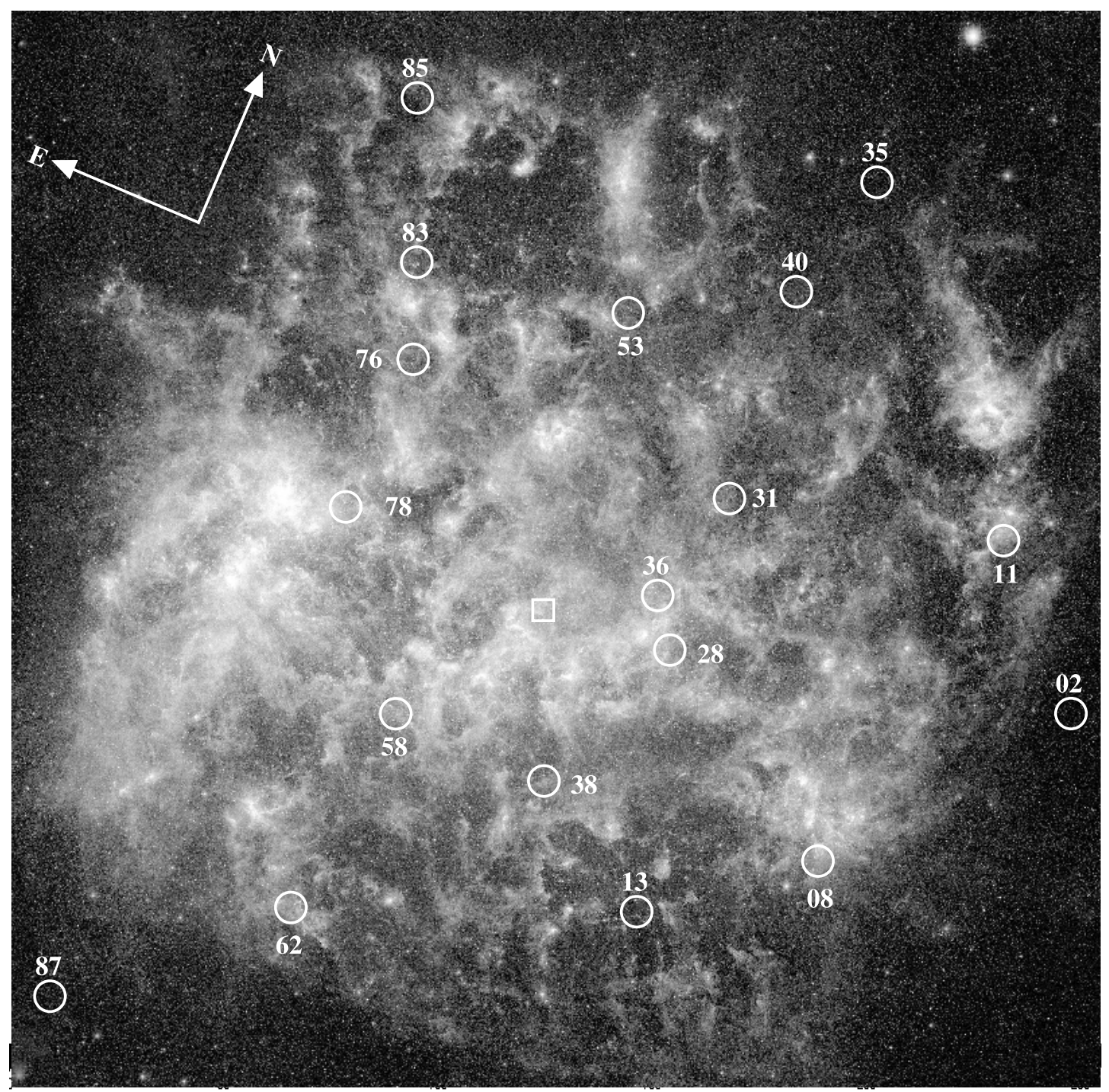

FIG. 1. - IRAC four-band mosaic of the LMC (SAGE) with the positions of the PNe overlaid on it (circles). The dynamical center of the LMC as given by Kim et al. (1998) is indicated by the square close to the center of the figure. [See the electronic edition of the Journal for a color version of this figure.]

line is always detected when the $18.7 \mu \mathrm{m}$ is present, but because the spectrum is noisier at the long-wavelength end of the LH module (see Fig. 2) we preferred to use the $18.71 \mu \mathrm{m}$ line flux for the abundance determination of this ion, which also has a larger transition probability and critical density. Similarly, we favored the use of the [Ne III] $15.55 \mu \mathrm{m}$ line instead of the $36.0 \mu \mathrm{m}$ line when both were measured. Table 2 also includes (mostly) upper limits on the argon line at $13.1 \mu \mathrm{m}$. Although not shown in this paper, the [Ar III] line at $8.99 \mu \mathrm{m}$ is detected in nine objects in the lowresolution spectra (J. Bernard-Salas et al., in preparation), and the [Ar II] $6.99 \mu \mathrm{m}$ line in two objects. The line fluxes were measured using the Gaussian line-fitting routine in Smart. These were derived for each nod independently. The uncertainty in the fluxes was assumed to be the largest of either the difference between the flux in the nod positions, or the uncertainty in the fit. These errors are given as footnotes in the table. The upper limits were calculated from a Gaussian fit with height 3 times the rms and a FWHM as given by the resolution of the instrument.

\subsection{Assumed Parameters}

Table 1 lists the $\mathrm{H} \beta$ flux, extinction, electron density $\left(N_{e}\right)$, and electron temperature $\left(T_{e}\right)$ assumed to calculate the abundances.
These values were compiled from a large list of references which are given in the footnote of the table. When several values were given by different authors an average was used. Only in the cases where several values differed by a large amount (i.e., SMP LMC 87, SMP SMC 11), we assumed those we estimate are more accurate. The infrared lines are little affected by uncertainties in the $T_{e}$ and extinction corrections but the abundances relative to hydrogen are derived using optical measurements of $\mathrm{H} \beta$, which are affected by these factors. The extinction law used is that of Fluks et al. (1994). In two cases no $N_{e}$ was reported and we assumed a value of $3000 \mathrm{~cm}^{-3}$, which seems a reasonable value in view of the other measurements.

\subsection{Abundance Determination}

Using the above parameters, the ionic abundances were computed from the infrared line intensities using equation (1) of Bernard-Salas et al. (2001). The results are shown in Table 3. The total abundances are also given in this table, where sometimes a correction due to missing ionization stages is necessary.

For the sulfur abundance the addition of $\mathrm{S}^{+}, \mathrm{S}^{+2}$, and $\mathrm{S}^{+3}$ is sufficient for those nebulae for which no $\mathrm{O}^{+3}$ is observed. The $\mathrm{S}^{+}$ abundance was determined using the optical lines of $\mathrm{S}^{+}$measured 


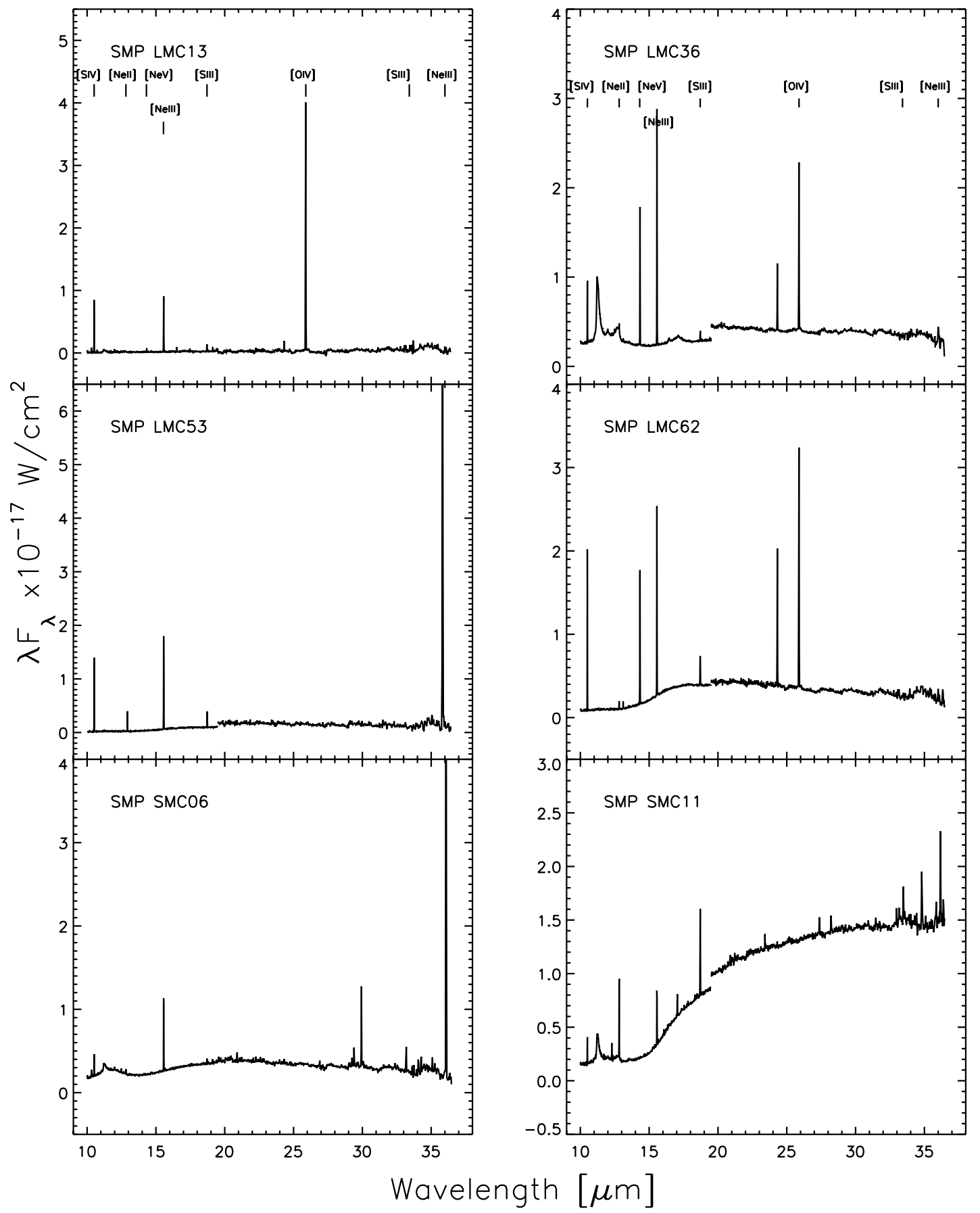

FIG. 2.- SH and LH spectra of a handful of PNe. The jump around $19.5 \mu \mathrm{m}$ is due to the larger background contribution that falls in the LH slit compared to the smaller SH slit (see $\S 2$ ).

by Meatheringham \& Dopita (1991a, 1991b) and Stanghellini et al. (2002, 2003). The contribution from this ion is usually small and on the order of what it is found in Galactic PNe. For the PNe which show the [O IV] line we must take into account the possibility that $\mathrm{S}^{+4}$ is present. An estimate may be obtained by looking at the two photoionization models of Me2-1 (Surendiranath et al. 2004) and NGC 6886 (Pottasch \& Surendiranath 2005). Both of these PNe are excited by high-temperature stars (with $T_{\text {eff }}$ between 140,000 and $180,000 \mathrm{~K}$ ) and both show [O IV] lines. In addition, Me2-1 has lower average abundances compared to Galactic PNe, which are closer to the nebulae studied here. Both models give similar results and show that $\mathrm{S}^{+4}$ contributes between $7 \%$ and $23 \%$ of the total sulfur abundance based on the $\mathrm{S}^{+4} / \mathrm{S}^{+3}$ ratio. We have used these numbers to correct the sulfur abundances in Table 3 .

In the case of neon no [Ne IV] line has been observed. For those $\mathrm{PNe}$ which do not show an [O IV] line it is unlikely that there is any $\mathrm{Ne}^{+3}$ because it requires a higher energy radiation field than does $\mathrm{O}^{+3}$. For those PNe which do show the [O IV] line (seven in the LMC and two in the SMC) a correction must be made. This can be done in two ways. First, using the same models as for sulfur we obtain a contribution of $\mathrm{Ne}^{+3}$ that varies from $2 \%$ to $33 \%$ of the total neon abundance depending on the strength of the $[\mathrm{Ne} \mathrm{v}]$ line. A second way of determining the correction could be done by looking at the neon abundances in the sample studied by Pottasch $\&$ Bernard-Salas (2006). This study made use of the same infrared 
[SIV]

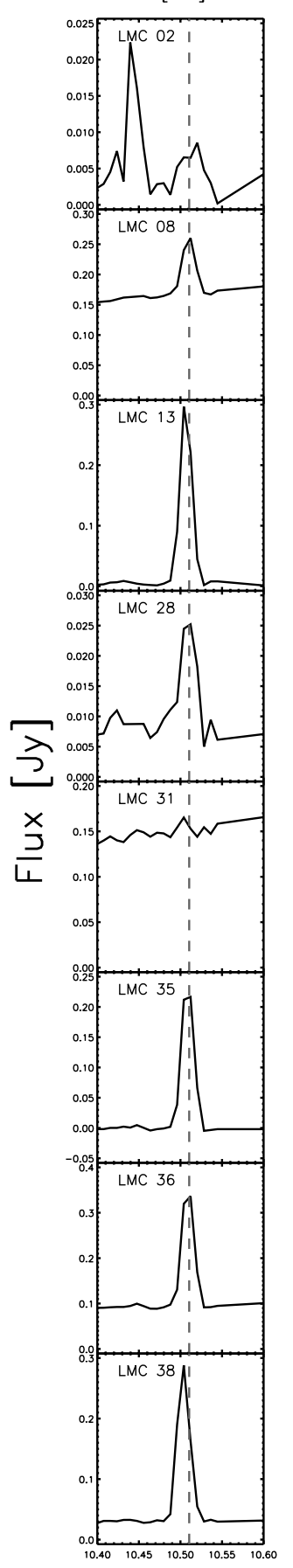

[Nell]

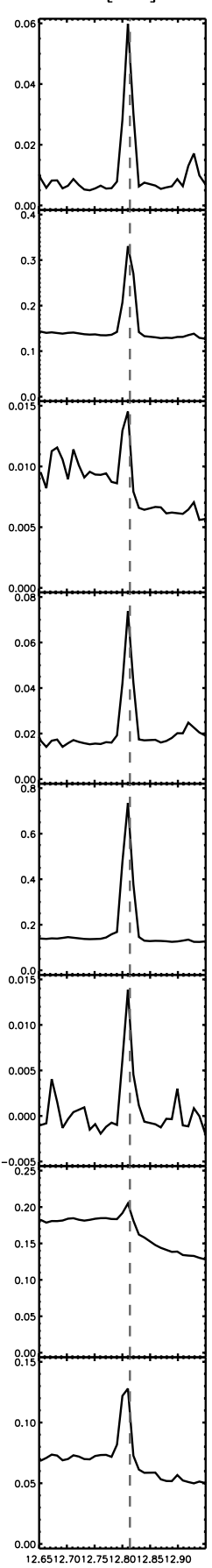

$[\mathrm{NeV}]$
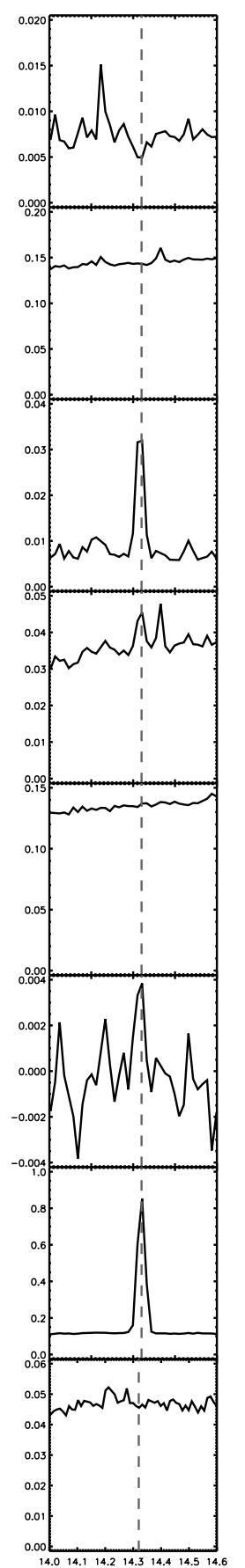

[Nelll]
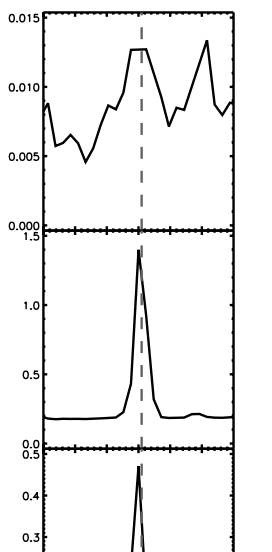
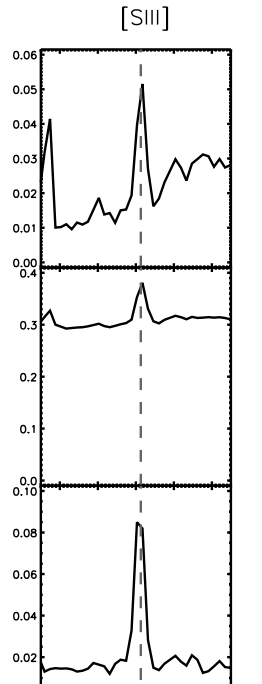

0
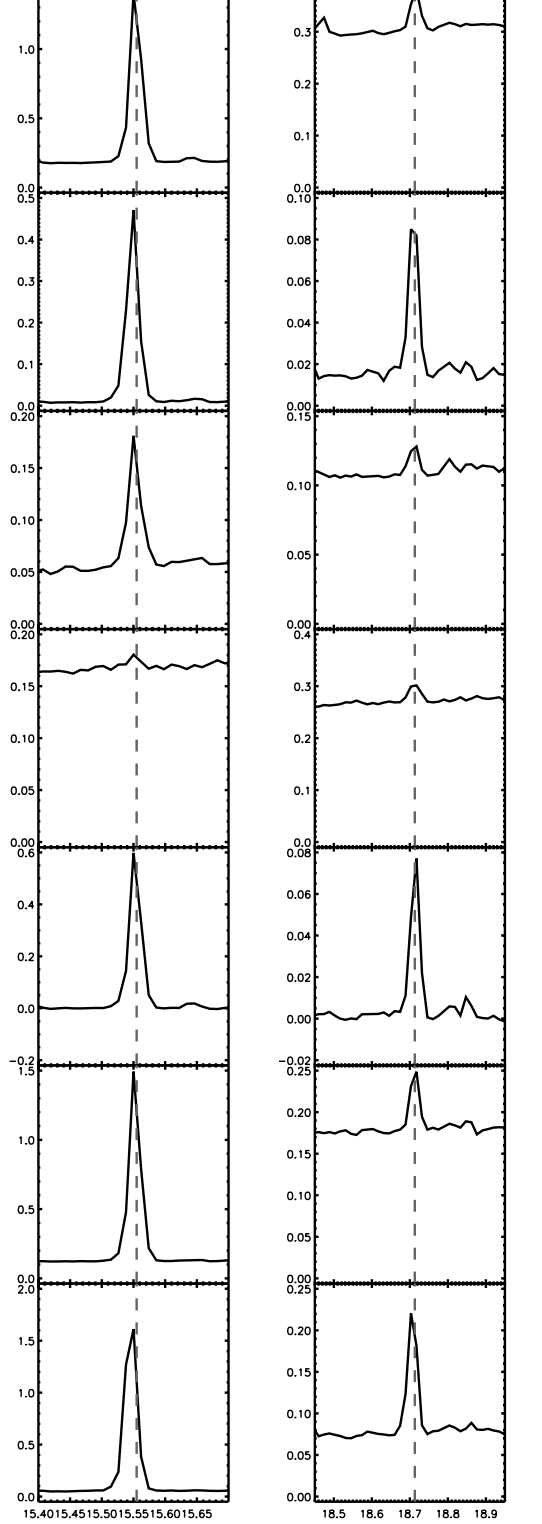

Wavelength $[\mu \mathrm{m}]$

FIG. 3.- Inset of the [S IV], [Ne II], [Ne v], [Ne III], and [S III] fine-structure lines for each object, except for SMP LMC 11, because this spectrum was shown in BernardSalas et al. (2006). The vertical dashed lines indicate the nominal position of the lines in the vacuum.

lines as used here and had both [Ne III] and [Ne v] lines, but the ultraviolet lines of [ $\mathrm{Ne}$ IV] at $2422 \AA$ were also observed so that $\mathrm{Ne}^{+3}$ could also be determined. For these $\mathrm{PNe}$ the $\mathrm{Ne}^{+3}$ abundance was on average $21 \%$ of the total neon abundance and $35 \%$ of the sum of $\mathrm{Ne}^{+2}$ and $\mathrm{Ne}^{+4}$. To be consistent with the sulfur abundance we have used the first method, but the results using the second method would result in the same values within the expected errors. The total neon abundances are given in Table 3 .

The uncertainty in the values of both the $\mathrm{Ne}^{+3}$ and $\mathrm{S}^{+4}$ abundances is probably not more than a factor of 2 . This leads to a maximum error for sulfur and neon of about $30 \%$ from this source. Uncertainties of measurement of the other ions in the infrared are about $10 \%$ with only a few exceptions (see footnote in Table 2 ). The error in the optical lines we used is of the same order. In addition, an error in the extinction affects the $\mathrm{H} \beta$ flux and uncertainties in the electron temperature dominate the uncertainty in our abundance determination. By comparing the optical measurements for the same objects by different observers in the literature, which usually agree, we estimate that the total error remains within $50 \%$ except for SMP LMC 08 and SMP SMC 11. The abundances 

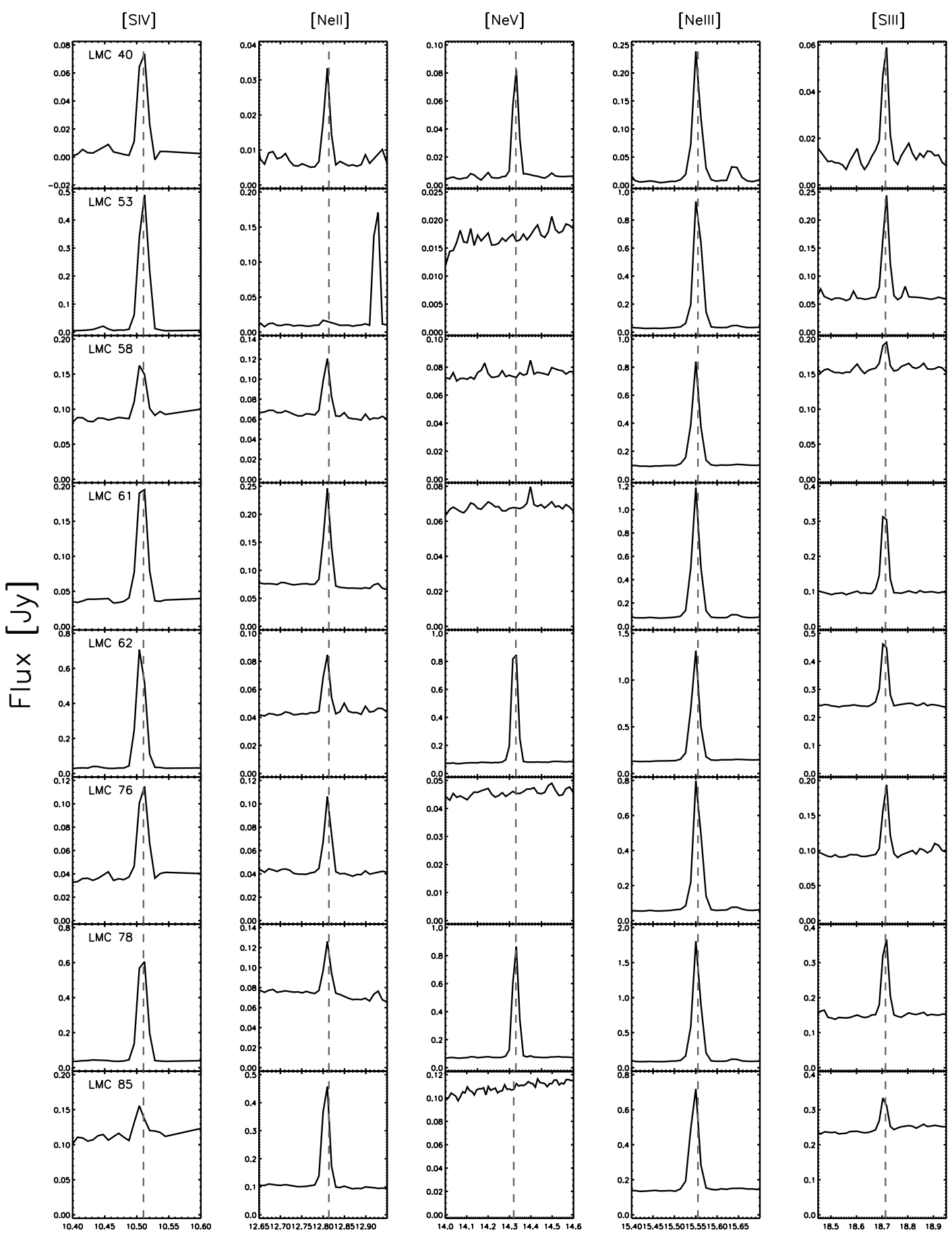

Wavelength $[\mu \mathrm{m}]$

FIG. 3-Continued

of SMP LMC 08 are uncertain, probably because of the assumed $\mathrm{H} \beta$ flux (see $\S 5.3$ ). In SMP SMC 11 the uncertainty is dominated by the inconsistent $\mathrm{H} \beta$ flux and extinction quoted in the literature and for the purpose of this paper the most recent values given in the literature have been adopted.

\section{COMPARISON SOURCES}

This section describes the sources to which the MC PN abundances are compared in $\S 5.2$. These comparison sources include
Galactic PNe and Galactic, MC, M33, and M83 H II regions, and the solar abundance.

\subsection{Solar Values}

The solar carbon, oxygen, sulfur, argon, and neon abundances have been subject to significant changes, especially during the last 7 years. These changes reflect in some way the difficulty in deriving solar abundances. The neon and argon abundances are especially troublesome because there are no lines of these elements in the 

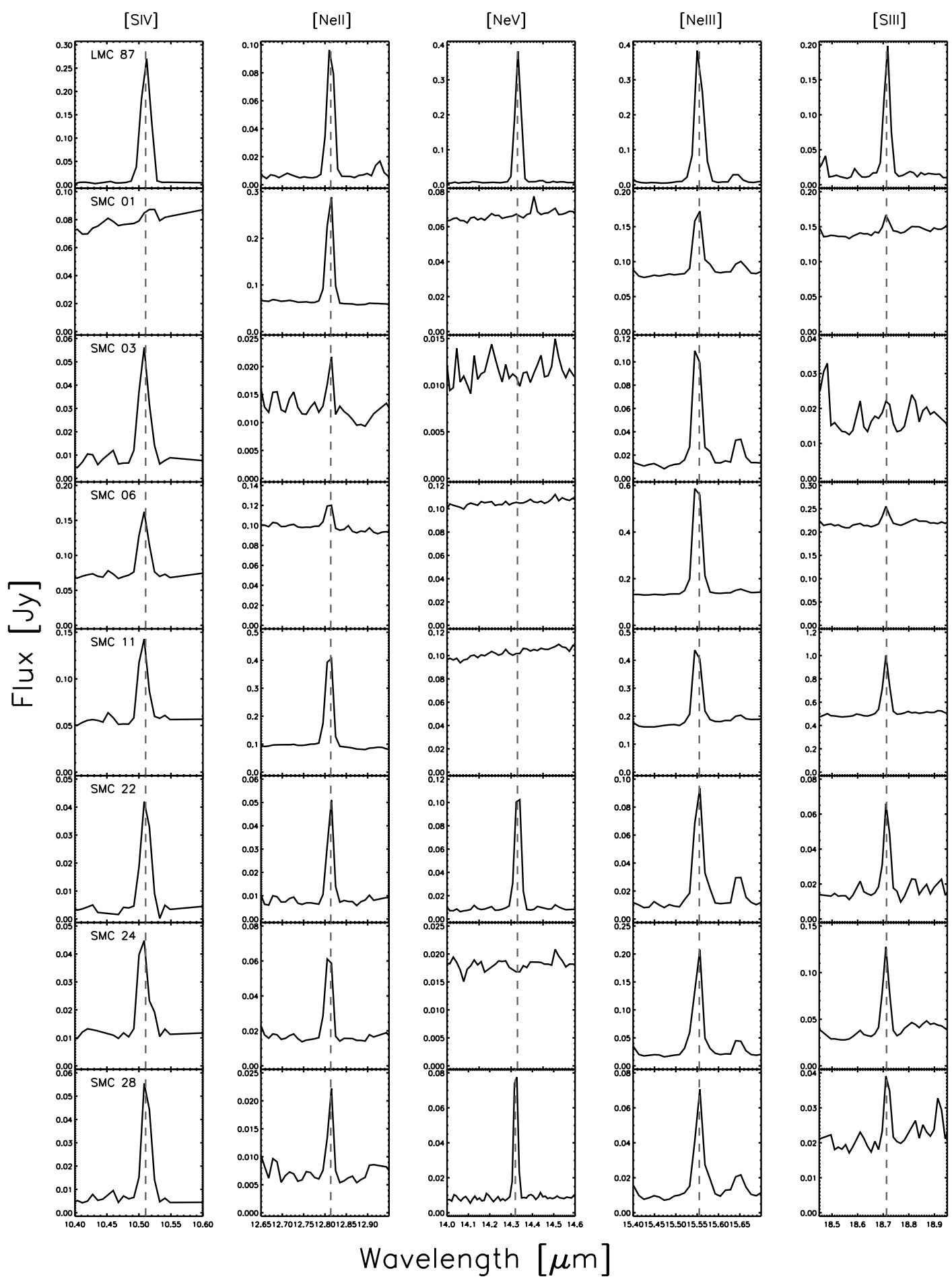

FIg. 3-Continued

solar photosphere and their abundances must be derived from coronal lines. Asplund et al. (2005) quoted a neon value of $6.9 \times 10^{-5}$ using the oxygen solar abundance and assuming a ratio of the $\mathrm{Ne} / \mathrm{O}$ of 0.15 . Previously Feldman \& Widing (2003), using coronal line measurements, found a neon abundance of $1.2 \times 10^{-4}$. This value is much higher than the value by Asplund et al. (2005) but agrees better with the earlier values reported by Grevesse \& Sauval (1998). This discrepancy is important in the debate over the consistency of the helioseismological measurements and the solar model (Antia \& Basu 2005; Bahcall et al. 2005). The neon abundance derived by Pottasch \& Bernard-Salas (2006) in a sample of Galactic PNe is more consistent with the higher neon value of Feldman \& Widing (2003). Very recently Landi et al. (2007) derived a value of $1.29 \times 10^{-4}$, again higher than the value given by Asplund et al. (2005) and in very good agreement with the previous values reported Feldman \& Widing (2003) and Grevesse $\&$ Sauval (1998). The quoted value of the solar sulfur abundance has been decreasing in the last few years. The sulfur abundance derived by Grevesse \& Noels (1993) is $1.4 \times 10^{-5}$, while Asplund et al. (2005) find $0.94 \times 10^{-5}$. Given these discrepancies, in the 
TABLE 2

Line Fluxes of the Observed LMC and SMC PNe

\begin{tabular}{|c|c|c|c|c|c|c|c|}
\hline Object & $\begin{array}{c}{[\mathrm{S} \text { Iv }]} \\
(10.51 \mu \mathrm{m})\end{array}$ & $\begin{array}{c}{[\mathrm{Ne} \mathrm{II}]} \\
(12.81 \mu \mathrm{m})\end{array}$ & $\begin{array}{c}{[\mathrm{Ar} \mathrm{v}]} \\
(13.10 \mu \mathrm{m})\end{array}$ & $\begin{array}{c}{[\mathrm{Ne} v]} \\
(14.31 \mu \mathrm{m})\end{array}$ & $\begin{array}{c}{[\mathrm{Ne} \text { III] }} \\
(15.55 \mu \mathrm{m})\end{array}$ & $\begin{array}{c}{[\mathrm{S} \text { III] }} \\
(18.71 \mu \mathrm{m})\end{array}$ & $\begin{array}{c}{[\mathrm{O} \mathrm{IV}]} \\
(25.89 \mu \mathrm{m})\end{array}$ \\
\hline 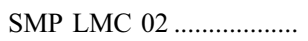 & $<0.72$ & 1.84 & $<0.32$ & $<0.26$ & $<0.66$ & 0.82 & $<0.75$ \\
\hline 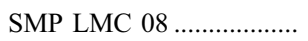 & 2.95 & 7.48 & $<0.38$ & $<0.38$ & 34.19 & 1.98 & $<0.63$ \\
\hline 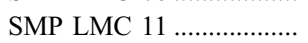 & $<1.08$ & 1.89 & $<0.77$ & $<0.69$ & 0.67 & $<0.68$ & $<1.38$ \\
\hline SMP LMC 13 & 12.08 & $0.22^{\mathrm{a}}$ & $<0.19$ & 0.94 & 12.74 & 2.10 & 55.54 \\
\hline SMP LMC 28 & 0.44 & 1.96 & $<0.30$ & 0.32 & 3.55 & 0.51 & $<1.15$ \\
\hline 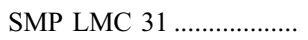 & $<0.75$ & 22.45 & $<0.31$ & $<0.17$ & $0.38^{\mathrm{a}}$ & 0.99 & $<1.09$ \\
\hline 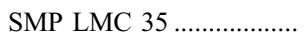 & 10.29 & 0.48 & $<0.19$ & $0.20^{\mathrm{a}}$ & 16.53 & 1.87 & 27.22 \\
\hline 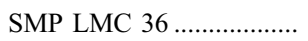 & $12.75^{\mathrm{a}}$ & 1.34 & 0.52 & 25.37 & 36.35 & 1.83 & $22.31^{\mathrm{a}}$ \\
\hline 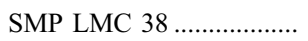 & $12.96^{\mathrm{a}}$ & 2.57 & $<0.24$ & $<0.31$ & 48.67 & 3.80 & $<1.53$ \\
\hline 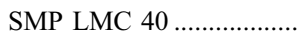 & 2.96 & 0.87 & $<0.24$ & 2.59 & 6.29 & 1.33 & 24.04 \\
\hline 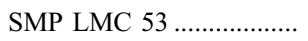 & 20.55 & 0.90 & $<0.23$ & $<0.26$ & 26.17 & 4.57 & $<1.49$ \\
\hline 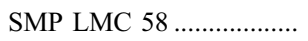 & 2.92 & 2.06 & $<0.24$ & $<0.38$ & 20.06 & 1.10 & $<2.16$ \\
\hline 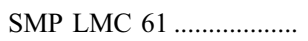 & 7.59 & 6.11 & $<0.46$ & $<0.35$ & 30.09 & 6.36 & $<0.59$ \\
\hline 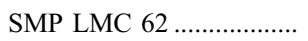 & 27.80 & 1.41 & 1.24 & 28.80 & 32.06 & 6.47 & 38.91 \\
\hline 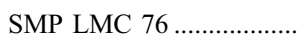 & 4.05 & 2.28 & $<0.29$ & $<0.23$ & 20.66 & 2.33 & $<0.42$ \\
\hline 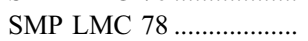 & 25.63 & 1.92 & 0.94 & 26.83 & 46.13 & 5.83 & 47.39 \\
\hline 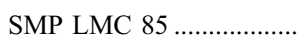 & 1.85 & 13.15 & $<0.27$ & $<0.40$ & 16.53 & 2.33 & $<0.86$ \\
\hline 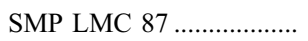 & 11.39 & 3.63 & 0.75 & 12.53 & 11.28 & 4.58 & 39.53 \\
\hline 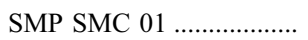 & $<0.53$ & 8.10 & $<0.29$ & $<0.34$ & 2.89 & 0.63 & $<0.66$ \\
\hline 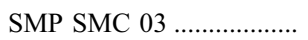 & 2.11 & $<0.35$ & $<0.32$ & $<0.24$ & 3.09 & $<0.48$ & $<1.25$ \\
\hline 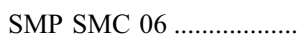 & 4.41 & 0.95 & $<0.23$ & $<0.36$ & 14.87 & 0.92 & $<0.65$ \\
\hline 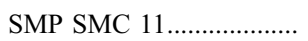 & 3.76 & 13.44 & $<0.30$ & $<0.37$ & 8.19 & 12.67 & $<1.18$ \\
\hline 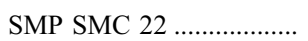 & 1.67 & 1.30 & $<0.30$ & 3.60 & 2.53 & $1.25^{\mathrm{a}}$ & 7.44 \\
\hline 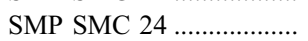 & 1.52 & 1.82 & $<0.33$ & $<0.25$ & 7.46 & 2.28 & $<0.84$ \\
\hline 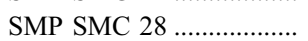 & $1.53^{\mathrm{a}}$ & 0.43 & $<0.24$ & 2.53 & 1.70 & 0.47 & $1.29^{\mathrm{a}}$ \\
\hline
\end{tabular}

Notes.-Fluxes in units of $\times 10^{-14} \mathrm{erg} \mathrm{cm}^{-2} \mathrm{~s}^{-1}$. Unless otherwise indicated, the uncertainties are less than $10 \%$ for all the lines except the [S Iv] line flux, which has an uncertainty between $10 \%$ and $20 \%$.

${ }^{a}$ These lines have uncertainties in the flux between $20 \%$ and $30 \%$, except for the [O Iv] line in SMP SMC 28 where the error is $42 \%$.

TABLE 3

Neon and Sulfur Abundances

\begin{tabular}{|c|c|c|c|c|c|c|c|c|c|c|c|}
\hline Object & $\mathrm{Ne}^{+} / \mathrm{H}$ & $\mathrm{Ne}^{+2} / \mathrm{H}$ & $\mathrm{Ne}^{+4} / \mathrm{H}$ & $\mathrm{ICF}(\mathrm{Ne})$ & $\mathrm{Ne} / \mathrm{H}$ & $\mathrm{S}^{+} / \mathrm{H}$ & $\mathrm{S}^{+2} / \mathrm{H}$ & $\mathrm{S}^{+3} / \mathrm{H}$ & $\operatorname{ICF}(\mathrm{S})$ & $\mathrm{S} / \mathrm{H}$ & $\mathrm{Ne} / \mathrm{S}$ \\
\hline SMP LMC $02 \ldots \ldots \ldots$. & 3.14 & $<0.55$ & $\ldots$ & 1 & 3.41 & $\ldots$ & 1.26 & $<0.22$ & 1 & 1.37 & 25 \\
\hline SMP LMC $08^{\mathrm{a}} \ldots \ldots \ldots$ & 32.10 & 73.60 & $\ldots$ & 1 & 105.70 & $\ldots$ & 9.06 & 2.64 & 1 & 11.70 & 90 \\
\hline SMP LMC $11 \ldots \ldots \ldots .$. & 4.56 & $\ldots$ & $\ldots$ & 1 & 4.56 & $\ldots$ & $\ldots$ & $\ldots$ & $\ldots$ & $\ldots$ & $\ldots$ \\
\hline SMP LMC $13 \ldots \ldots \ldots$ & 0.17 & 4.81 & 0.05 & 1.02 & 5.12 & 0.14 & 1.46 & 1.75 & 1.20 & 4.01 & 13 \\
\hline SMP LMC $28 \ldots \ldots \ldots . .$. & 4.68 & 4.11 & 0.45 & 1.09 & 10.06 & 0.79 & 1.06 & 0.19 & 1 & 2.03 & 49 \\
\hline SMP LMC $31 \ldots \ldots \ldots$ & 6.95 & 0.06 & $\ldots$ & 1 & 7.01 & 0.13 & 0.34 & $<0.05$ & 1 & 0.47 & 148 \\
\hline SMP LMC $35 \ldots \ldots \ldots . .$. & 0.35 & 5.86 & $\ldots$ & 1 & 6.21 & 0.11 & 1.10 & 1.28 & 1.19 & 2.97 & 21 \\
\hline SMP LMC $36 \ldots \ldots \ldots . .$. & 0.32 & 4.36 & 0.46 & 1.18 & 5.96 & $\ldots$ & 0.38 & 0.58 & 1.23 & 1.18 & 51 \\
\hline SMP LMC $38 \ldots \ldots \ldots .$. & 0.84 & 8.25 & $\ldots$ & 1 & 9.09 & 0.29 & 1.62 & 1.04 & 1 & 2.95 & 31 \\
\hline SMP LMC $40 \ldots \ldots \ldots . .$. & 1.19 & 4.19 & 0.24 & 1.08 & 6.06 & 0.53 & 1.45 & 0.68 & 1.10 & 2.92 & 21 \\
\hline SMP LMC $53 \ldots \ldots \ldots . .$. & 0.34 & 4.95 & $\ldots$ & 1 & 5.29 & 0.29 & 1.61 & 1.51 & 1 & 3.41 & 16 \\
\hline SMP LMC $58 \ldots \ldots \ldots . .$. & 0.74 & 3.95 & $\ldots$ & 1 & 4.69 & 0.05 & 0.79 & 0.35 & 1 & 1.18 & 40 \\
\hline SMP LMC $61 \ldots \ldots \ldots . .$. & 1.54 & 4.34 & $\ldots$ & 1 & 5.88 & 0.79 & 3.99 & 0.75 & 1 & 5.53 & 11 \\
\hline SMP LMC $62 \ldots \ldots \ldots .$. & 0.27 & 3.11 & 0.44 & 1.23 & 4.61 & 0.22 & 1.15 & 1.22 & 1.18 & 3.05 & 15 \\
\hline SMP LMC $76 \ldots \ldots \ldots . .$. & 0.48 & 2.33 & $\ldots$ & 1 & 2.81 & 0.19 & 0.79 & 0.24 & 1 & 1.22 & 23 \\
\hline SMP LMC $78 \ldots \ldots \ldots$ & 0.57 & 6.87 & 0.60 & 1.14 & 9.11 & 0.17 & 1.63 & 1.50 & 1.17 & 3.87 & 24 \\
\hline SMP LMC $85 \ldots \ldots \ldots$ & 2.70 & 1.98 & $\ldots$ & 1 & 4.68 & 0.40 & 1.37 & 0.17 & 1 & 1.94 & 24 \\
\hline SMP LMC $87 \ldots \ldots \ldots$ & 1.59 & 2.45 & 0.46 & 1.20 & 5.32 & 0.49 & 1.55 & 0.90 & 1.12 & 3.28 & 16 \\
\hline$\langle\mathrm{LMC}\rangle^{\mathrm{b}}$ & $\ldots$ & . & $\ldots$ & 然 & 6.03 & & . & & & 2.72 & 22 \\
\hline SMP SMC $01 \ldots \ldots \ldots$. & 3.97 & 0.73 & $\ldots$ & 1 & 4.70 & 0.04 & 0.41 & $<0.06$ & 1 & 0.46 & 103 \\
\hline SMP SMC $03 \ldots \ldots \ldots . .$. & $<0.58$ & 2.58 & $\ldots$ & 1 & 2.87 & 0.18 & $<0.35$ & 0.71 & 1 & 1.07 & 29 \\
\hline SMP SMC $06 \ldots \ldots \ldots .$. & 0.30 & 2.48 & $\ldots$ & 1 & 2.78 & 0.07 & 0.44 & 0.39 & 1 & 0.90 & 31 \\
\hline SMP SMC $11^{\mathrm{a}} \ldots \ldots \ldots$ & 1.62 & 0.48 & $\ldots$ & 1 & 2.10 & 0.01 & 1.16 & 0.08 & 1 & 1.25 & 17 \\
\hline SMP SMC $22 \ldots \ldots \ldots . .$. & 0.76 & 0.74 & 0.17 & 1.21 & 1.98 & 0.23 & 0.58 & 0.18 & 1.07 & 1.06 & 19 \\
\hline SMP SMC $24 \ldots \ldots \ldots .$. & 0.94 & 1.87 & $\ldots$ & 1 & 2.81 & 0.08 & 0.95 & 0.13 & 1 & 1.17 & 24 \\
\hline SMP SMC $28 \ldots \ldots \ldots . .$. & 0.37 & 0.76 & 0.21 & 1.33 & 1.71 & 0.23 & 0.45 & 0.31 & 1.12 & 1.10 & 16 \\
\hline$\langle\mathrm{SMC}\rangle \ldots \ldots \ldots \ldots \ldots$ & $\ldots$ & $\ldots$ & $\ldots$ & $\ldots$ & 2.71 & $\ldots$ & $\ldots$ & $\ldots$ & $\ldots$ & 1.00 & 27 \\
\hline
\end{tabular}

Note.-The neon and sulfur abundances are in $\times 10^{-5}$ and $\times 10^{-6}$, respectively.

a Large uncertainty in the abundance (see $\S 3.3$ ).

b The average also includes SMP LMC 83 from Bernard-Salas et al. (2004). 
rest of the paper instead of assuming a certain value we will refer and compare to the above range of solar values.

\subsection{PNe and $\mathrm{H}$ II Regions}

For comparison purposes we have selected a sample of PNe and $\mathrm{H}$ in regions for which abundances were also derived from infrared data and in a similar way to the PNe presented in this paper. The Galactic PNe abundances in Pottasch \& Bernard-Salas (2006) using ISO data have been complemented with the Spitzer-derived abundances of IC 2448 (Guiles et al. 2007), M1-42 (Pottasch et al. 2007), and NGC 2392 (S. R. Pottasch et al., in preparation). Galactic and $\mathrm{MC} \mathrm{H}$ II regions were taken from Martín-Hernández et al. (2002) and Vermeij \& van der Hulst (2002), respectively. They include $I S O$-derived abundances from $26 \mathrm{H}$ II regions in the Milky Way, 13 in the LMC, and three in the SMC. The Spitzer abundances in Lebouteiller et al. (2007) of the giant $\mathrm{H}$ II regions NGC 3603 (in the Milky Way), 30 Doradus (LMC), and NGC 346 (SMC) are also included. While Lebouteiller et al. (2007) derive abundances at several positions in each region, the calculated abundances are very similar for a given region and here we adopt their average value. Rubin et al. (2007) recently derived the Ne/S abundance ratio of $\mathrm{H}$ II regions in M83 using Spitzer data, but the absolute values are not given. The same authors are working on a study of $\mathrm{H}$ II regions in M33 and we use their neon and sulfur abundance ranges ${ }^{5}$ in Figure 5.

\section{DISCUSSION}

\subsection{Neon, Sulfur, and the Ne/S Ratio}

Neon and sulfur are alpha-process elements and therefore should track each other. Their abundances are representative of the chemical composition of the cloud from which they formed. Recent work has suggested that some PNe may produce neon in the course of evolution (Marigo et al. 2003). Leisy \& Dennefeld (2006) claim that, because their derived oxygen and neon abundances correlate with each other, when oxygen is enriched neon must be also self-enriched in their MC sample. According to the theoretical models of Karakas \& Lattanzio (2003) this enrichment is modest and the mass range at which neon production in low- and intermediate-mass stars may occur is very small (around $\left.3 M_{\odot}\right)$. Thus statistically few PNe should experience such an effect.

From the LMC abundances listed in Table 3, SMP LMC 08 shows unusually high abundances of neon and sulfur. Except for SMP LMC 11, this PN has the lowest $\mathrm{H} \beta$ flux of the LMC sample (see Table 1), and it is likely that the high abundances are in part the result of this low $\mathrm{H} \beta$ flux which we use to derive the abundance. The neon enrichment predicted by Karakas $\&$ Lattanzio (2003) and Marigo et al. (2003) is not large enough to predict such high values. The lowest neon abundance corresponds to SMP LMC 76, which also has a low sulfur abundance compared to the rest of the objects (but not the lowest). The neon abundance of SMP LMC 11 has been derived using only the $\mathrm{Ne}^{+}$ stage of ionization. This is a very low excitation object, and has been described as a preplanetary nebula by Bernard-Salas et al. (2006) due to its very peculiar infrared spectrum which shows, among other features, molecular absorption bands of acetylene and polyacetylene. There are no $[\mathrm{Ne}$ III] or $[\mathrm{S}$ III] and [S IV] lines in the IRS spectrum.

Not including SMP LMC 08, the average neon abundance of the $\mathrm{PNe}$ in the $\mathrm{LMC}$ is $6.0 \times 10^{-5}$, which is $1 / 2.7$ of the average

\footnotetext{
5 These abundances were presented in the Xiang Shan workshop in 2007 (http://ast.pku.edu.cn/ xs2007).
}

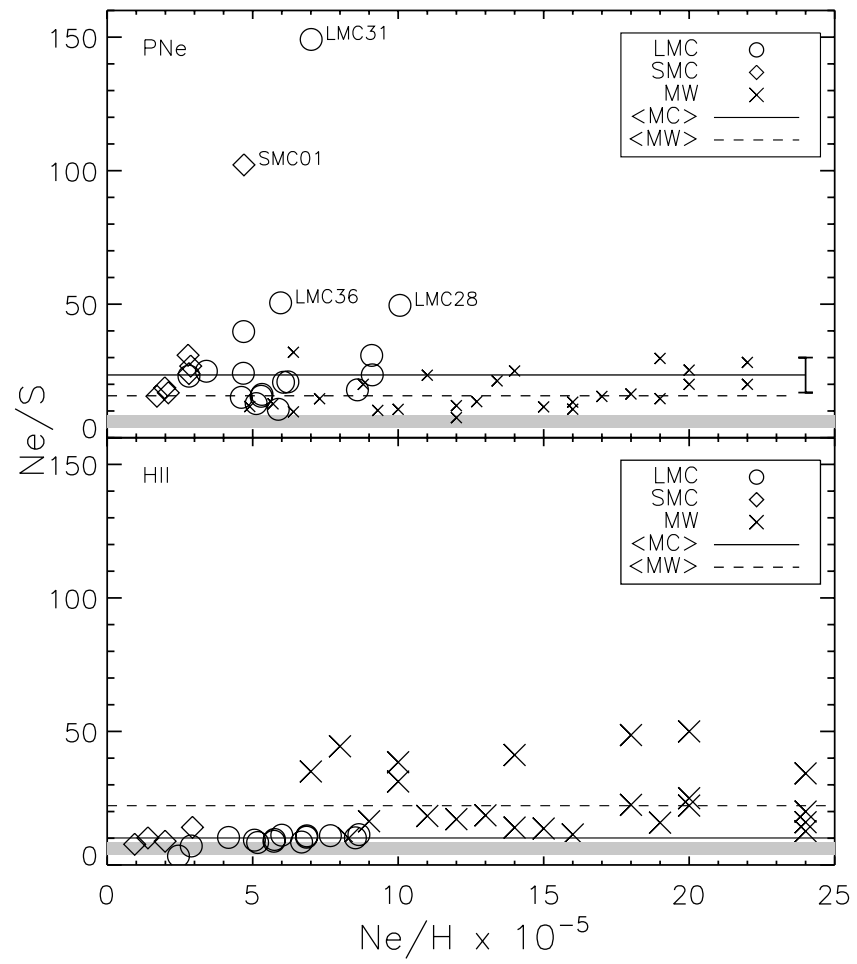

FIG. 4.- Ne/S ratio with respect to the total neon abundance of the PNe (top) and $\mathrm{H}$ II regions (bottom). The gray band represents the solar $\mathrm{Ne} / \mathrm{S}$ ratio. The horizontal lines represent the median of the $\mathrm{Ne} / \mathrm{S}$ for $\mathrm{PNe}$ (with a typical error bar on the right side of the line) and $\mathrm{H}$ II regions in the Galaxy, the LMC, and the SMC.

neon abundance of Galactic PNe $\left(1.6 \times 10^{-4}\right)$ used for comparison $(\S 4.2)$. The mean sulfur abundance is $2.7 \times 10^{-6}$, which yields a slightly lower ratio of $1 / 3.7$ when compared to the average value of Galactic PNe $\left(0.99 \times 10^{-5}\right)$. The SMC sample contains only seven objects. Although this is a small number with which to attempt statistics, it can be seen in Table 3 that the sulfur and especially the neon abundances are very similar in most of the SMC objects. The mean neon abundance of the SMC PNe is $2.7 \times 10^{-5}$, which is $1 / 6.0$ of the average neon abundance in Galactic PNe. The sulfur abundance in the SMC PNe is also low, $1 / 10$ of the average Galactic PNe sulfur abundance. Thus, keeping in mind that the SMC sample contains only a few objects, it seems that either the sulfur abundance in MC PNe is lower than in Galactic PNe or the neon abundance is higher.

Figure 4 is a plot of the $\mathrm{Ne} / \mathrm{S}$ ratio against the neon abundance (as an indicator of metallicity) for PNe (top) and $\mathrm{H}$ II regions (bottom). This abundance ratio has the advantage over the elemental abundance measurements in that any uncertainty introduced by combining infrared measurements with optical hydrogen measurements cancels out, and uncertainties in $T_{e}$ are much reduced. Also plotted are the median values for the different data sets. The figure shows that most of the MC PNe have ratios between 15 and 30 (with a median value of 23.5), with SMC and LMC PNe displaying a similar range of values. This range is similar to the ratio displayed by Galactic PNe (7.5-32), although as we saw before the MC PNe have a slightly higher ratio (solid and dashed lines). The $\mathrm{Ne} / \mathrm{S}$ ratio in Galactic $\mathrm{H}$ II regions ranges from 10 to 50 with a median value of 21 (Fig. 4, dashed line) and agrees very well with the MC PNe. The MC H II regions have a lower $\mathrm{Ne} / \mathrm{S}$ ratio on average, but the number of sources is not very large. The H II regions studied by Rubin et al. (2007) in the metal-rich galaxy M83 have Ne/S ratios that vary from 24.4 to 41.9 and thus are similar to the ratios shown by the MC PNe. In summary, the 
$\mathrm{Ne} / \mathrm{S}$ ratios show large variations within each data set. The fact that most LMC/SMC PNe show values which are similar to Galactic $\mathrm{PNe}$ and different $\mathrm{H}$ II regions implies that these elements have a common origin and if any enrichment of neon has occurred it has remained modest. This agrees with the result of Dopita et al. (1997), who find no sign of dredge-up of ${ }^{22} \mathrm{Ne}$ in their sample of LMC PNe.

There are two $\mathrm{PNe}$ with a $\mathrm{Ne} / \mathrm{S}$ ratio which is significantly higher than any of the comparison data sets. Two other objects have a ratio of $\sim 50$, which is high compared to the rest of the sample of $\mathrm{PNe}$ although some Galactic $\mathrm{H}$ in regions also reach such values. These objects are labeled in Figure 4 (top). The extremely high values of the Ne/S ratio in SMP LMC 31 and SMP SMC 01 are mainly the result of their lower sulfur abundance. These two objects show the lowest sulfur abundances: SMP LMC 31 has a sulfur abundance which is 5.7 times lower than the average LMC $\mathrm{PNe}$, and the sulfur abundance in SMP SMC 01 is about 2.2 lower than the average SMC PNe (see Table 3). These differences in sulfur can account for the high ratio observed in these two objects. However, these two objects together with SMP LMC 28 also show a high neon abundance compared to the rest of objects in their sample and it is therefore possible that neon enrichment has taken place in these objects.

The gray band in Figure 4 indicates the lower and higher solar ratio found using the abundances given in $\S 3.1$ and it is clearly lower than Galactic and $\mathrm{MC} \mathrm{PNe}$ or $\mathrm{H}$ II regions. This is known and several authors have already discussed the idea that the solar sulfur abundance seems too high compared to Galactic PNe and H II regions (Pottasch \& Bernard-Salas 2006; Marigo et al. 2003; Martín-Hernández et al. 2002; Henry et al. 2004). In addition, several authors (Pottasch \& Bernard-Salas 2006; Wang \& Liu 2007) favor the higher neon solar abundance given by Feldman \& Widing (2003) and Landi et al. (2007) instead of the one quoted by Asplund et al. (2005). This work also supports the higher neon abundance in the literature, but from Figure 4 it is clear that the Ne/S in most data sets is higher than the solar ratio.

\subsection{Comparison to Other Sources}

Figure 5 shows a comparison of the neon and sulfur abundances in PNe and $\mathrm{H}$ II regions in the Milky Way, LMC, SMC, and M33. The gray band in the figure represents the range of solar values found in the literature.

Galactic $\mathrm{PNe}$ and $\mathrm{H}$ II regions display a neon abundance that is closer to the higher solar value. Note that while we are comparing with solar values the effect of the Galactic abundance gradient has not been taken into account in this plot. The Galactic sources also show a clear underabundance of sulfur compared to the solar value. The neon abundances in $\mathrm{PNe}$ and $\mathrm{H}$ II regions show a remarkable agreement in the Milky Way, LMC, and SMC. M33, which is usually regarded as having half the solar metallicity (Magrini et al. 2007), shows a very large range of neon abundance which encompasses both the LMC and SMC PNe sample.

The interpretation of the sulfur abundance is complicated. As mentioned before, both Galactic $\mathrm{PNe}$ and $\mathrm{H}$ in regions show clearly an underabundance of sulfur with respect to solar, and this has been ascribed in some studies to the solar sulfur abundance being too high. The LMC PNe sulfur abundance shows a very large dispersion in values. Most PNe show sulfur values that are in the range of the LMC H II regions, but six objects are below that range. This does not mean that these PNe have anomalous sulfur abundances, as the number of LMC H II regions used for comparison is small, but it seems to point to a slightly lower sulfur abundance in the PNe. The low sulfur abundances shown by these six PNe are not due to errors in the ICF used to correct for $\mathrm{S}^{+4}$ because

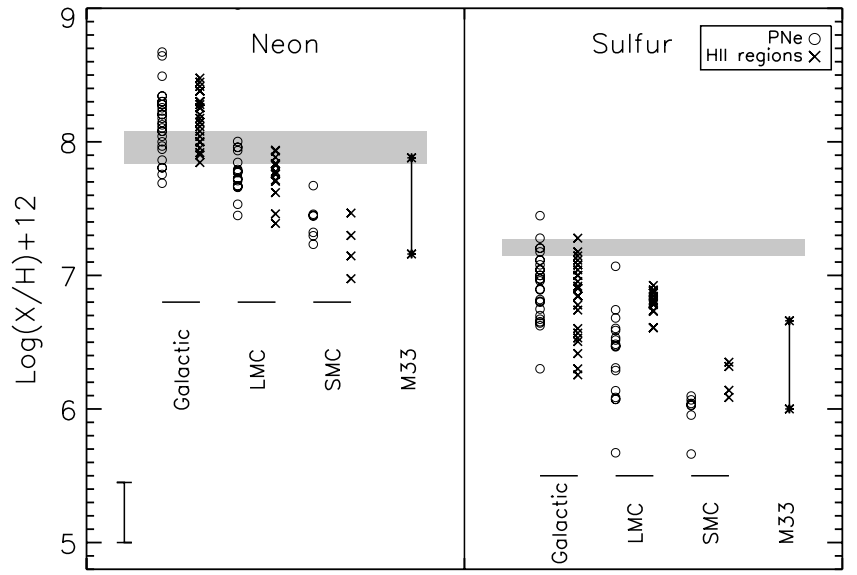

FIG. 5.-Comparison of the neon and sulfur abundances in $\mathrm{PNe}$ and $\mathrm{H}$ II regions for the Milky Way, the LMC, and the SMC. The range of abundances for $\mathrm{H}$ II regions in M33 are also shown. A $50 \%$ error bar for the LMC and SMC PNe is shown in the lower side of the figure ( $\operatorname{see} \S 3.3$ ).

some of them are in fact low-ionization PNe and no ICF is needed. We have compared the average abundance of sulfur in high- and low-excitation PNe separately and find similar average abundances of $2.9 \times 10^{-6}$ and $2.3 \times 10^{-6}$ for the high- and low-excitation PNe, respectively. Except for SMP LMC 31, the range of sulfur abundances in the $\mathrm{H}$ II regions in M33 (which has a metallicity close to that of the LMC) is similar to the sulfur abundances we measure in these $\mathrm{PNe}$. The comparison of the $\mathrm{PNe}$ and $\mathrm{H}$ in region sulfur abundances in the SMC is hampered by the low number of sources. Considering the uncertainties both data sets compare well, although one source (SMP SMC 01) has a sulfur abundance which is clearly lower than the rest of $\mathrm{PNe}$ and $\mathrm{H}$ II regions. We do not know the reasons for the apparent sulfur depletion in SMP LMC 31 and SMP SMC 01. Sulfur can be depleted onto dust (e.g., MgS, FeS). Although both SMP LMC 31 and SMP SMC 01 do show a strong $\mathrm{MgS}$ feature, a further investigation of this possibility must be made before any conclusion can be drawn.

\subsection{Comparison with the Literature}

A comparison of the abundances derived in this paper with those derived in Dopita \& Meatheringham (1991a, 1991b) and Dopita et al. (1997) using photoionization models and Leisy \& Dennefeld (2006) using optical data is given in Table 4.

The neon abundances we derive are in agreement with the abundances of Dopita \& Meatheringham (1991a, 1991b). In most of the cases the agreement is very good. For the few objects where the agreement is not that satisfactory, the abundances usually compare well within a factor of 2 , which is reasonable considering the uncertainties in the abundances we derive and those involved in the use of photoionization models. Dopita et al. (1997) revised the abundances for eight LMC PNe adding HST spectroscopy to their ground observations. Some of their revised abundances agree with their previous determinations, but in some cases (especially for sulfur) the differences amount to a factor of 1.3-3. Our abundances are roughly similar to those of Leisy \& Dennefeld (2006) for most of the high-excitation $\mathrm{PNe}^{6}$ except for SMP LMC 62 and SMP LMC 83. Leisy \& Dennefeld (2006), however, state that their neon abundance for these two objects was poorly determined. For most of the remaining sources (low-excitation PNe) our abundances are significantly larger than those by Leisy \&

\footnotetext{
6 Those PNe showing high-excitation lines such as Ne v. See footnote in Table 4.
} 
TABLE 4

Abundance Comparison

\begin{tabular}{|c|c|c|c|c|c|c|c|c|}
\hline \multirow{2}{*}{$\begin{array}{l}\text { ОвJеCт } \\
(\mathrm{SMP})\end{array}$} & \multicolumn{4}{|c|}{$\mathrm{Ne} / \mathrm{H} \times 10^{-5}$} & \multicolumn{4}{|c|}{$\mathrm{S} / \mathrm{H} \times 10^{-6}$} \\
\hline & Present & $\mathrm{DM}^{\mathrm{a}}$ & $\mathrm{D} 97^{\mathrm{a}}$ & $\mathrm{LD}^{\mathrm{a}}$ & Present & $\mathrm{DM}^{\mathrm{a}}$ & $\mathrm{D} 97^{\mathrm{a}}$ & $\mathrm{LD}^{\mathrm{a}}$ \\
\hline 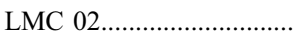 & 3.41 & $\ldots$ & 2.8 & $<0.18$ & 1.4 & 1.6 & 4.0 & $<4.2$ \\
\hline 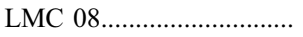 & 105.7 & 2.2 & 2.1 & 1.82 & 11.7 & 6.0 & 4.9 & 1109.7 \\
\hline 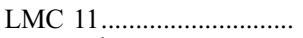 & 4.6 & $\ldots$ & $\ldots$ & 0.55 & $\ldots$ & $\ldots$ & $\ldots$ & 1.15 \\
\hline LMC $13^{\mathrm{b}} \ldots \ldots \ldots \ldots \ldots \ldots \ldots \ldots \ldots \ldots \ldots \ldots \ldots$ & 5.1 & 3.8 & $\ldots$ & 4.0 & 4.0 & 3.9 & $\ldots$ & $\ldots$ \\
\hline LMC $28^{\mathrm{b}} \ldots$ & 10.1 & $\ldots$ & $\ldots$ & $\ldots$ & 2.0 & $\ldots$ & $\ldots$ & $\ldots$ \\
\hline 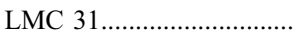 & 7.0 & $\ldots$ & $\ldots$ & 0.04 & 0.47 & $\ldots$ & $\ldots$ & 1.86 \\
\hline 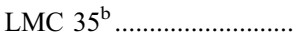 & 6.2 & 4.0 & 3.0 & 3.0 & 3.0 & 2.8 & 6.0 & 24.0 \\
\hline LMC $36^{\mathrm{b}} \ldots \ldots \ldots \ldots \ldots \ldots \ldots \ldots \ldots \ldots \ldots \ldots$ & 6.0 & $\ldots$ & $\ldots$ & 5.3 & 1.18 & $\ldots$ & $\ldots$ & $\ldots$ \\
\hline 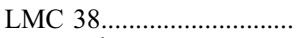 & 9.1 & 4.1 & $\ldots$ & 3.6 & 2.9 & 1 & $\ldots$ & 23.4 \\
\hline LMC $40^{\mathrm{b}} \ldots \ldots \ldots \ldots \ldots \ldots \ldots \ldots \ldots \ldots \ldots \ldots \ldots$ & 6.1 & 6.0 & 4.5 & 6.9 & 2.9 & 4.6 & 6.2 & 36.3 \\
\hline 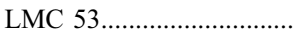 & 5.3 & $\ldots$ & $\ldots$ & 4.1 & 3.4 & $\ldots$ & $\ldots$ & 18.2 \\
\hline 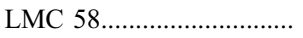 & 4.7 & 2.0 & $\ldots$ & 1.23 & 1.18 & 3.1 & $\ldots$ & 95.5 \\
\hline 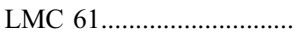 & 5.9 & 4.2 & $\ldots$ & 5.8 & 5.5 & 7.4 & $\ldots$ & 5.7 \\
\hline $\mathrm{LMC} 62^{\mathrm{b}} \ldots \ldots$ & 4.6 & 3.3 & $\ldots$ & 2.2 & 3.1 & 8.0 & $\ldots$ & 4.6 \\
\hline 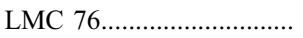 & 2.8 & 2.4 & 2.2 & 1.86 & 1.2 & 4.0 & 4.0 & $\ldots$ \\
\hline 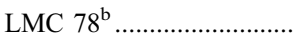 & 9.1 & 4.5 & $\ldots$ & 3.6 & 3.9 & 5.2 & $\ldots$ & 30.9 \\
\hline 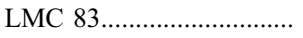 & 8.6 & 4.1 & 5.1 & $\ldots$ & 4.8 & 6.5 & 2.4 & $\ldots$ \\
\hline 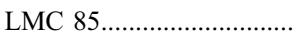 & 4.7 & 4.0 & 3.0 & 0.68 & 1.9 & 4.0 & 2.0 & 4.7 \\
\hline LMC $87^{\mathrm{b}} \ldots \ldots \ldots \ldots \ldots \ldots \ldots \ldots \ldots \ldots \ldots \ldots$ & 5.3 & 11.0 & $\ldots$ & 3.1 & 3.3 & 6.8 & $\ldots$ & 18.2 \\
\hline 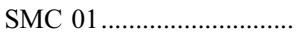 & 4.7 & 0.7 & $\ldots$ & 0.26 & 0.46 & $\ldots$ & $\ldots$ & $\ldots$ \\
\hline 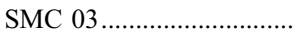 & 2.9 & 1.3 & $\ldots$ & 1.00 & 1.01 & $\ldots$ & $\ldots$ & 5.7 \\
\hline 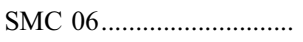 & 2.8 & 2.2 & $\ldots$ & 1.38 & 0.90 & 6.0 & $\ldots$ & 23.4 \\
\hline 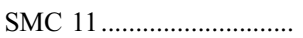 & 2.1 & 1.5 & $\ldots$ & $<1.00$ & 1.25 & 2.8 & $\ldots$ & $<5.9$ \\
\hline SMC $22^{\mathrm{b}} \ldots \ldots \ldots \ldots \ldots \ldots \ldots \ldots \ldots \ldots \ldots \ldots \ldots \ldots$ & 2.0 & 2.1 & $\ldots$ & 0.56 & 1.06 & 3.2 & $\ldots$ & 4.0 \\
\hline 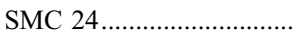 & 2.8 & $\ldots$ & $\ldots$ & 1.15 & 1.17 & $\ldots$ & $\ldots$ & 0.31 \\
\hline 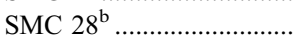 & 1.7 & 3.0 & $\ldots$ & 0.72 & 1.10 & 4.0 & $\ldots$ & 7.4 \\
\hline
\end{tabular}

a References to abundances: DM (Meatheringham \& Dopita 1991a, 1991b), D97 (Dopita et al. 1997), LD (Leisy \& Dennefeld 2006).

${ }^{\mathrm{b}}$ High-excitation PNe.

Dennefeld (2006). To determine the reasons for this discrepancy, we have calculated the $\mathrm{Ne}^{+2}$ abundance using their measured optical line at $3869 \AA$ and assumed $T_{e}$. The $\mathrm{Ne}^{+2}$ fractional abundance we derive using the optical line agrees within $30 \%$ with the results from the infrared line for the high-excitation $\mathrm{PNe}$ and a few of the low-excitation PNe (SMP LMC 53, SMP LMC 61, SMP LMC 76). For the rest of the objects the difference in the $\mathrm{Ne}^{+2}$ ionic abundance is large enough to account for the difference in the total neon abundance. A possible explanation for this discrepancy is the uncertainty of $T_{e}$ when deriving abundances using optical lines. In some PNe the $T_{e}$ derived using the $\mathrm{Ne}$ III is significantly lower than using other ions (Bernard-Salas et al. 2001). A lower $T_{e}$ increases the ionic abundance, and a difference of a couple of thousand degrees Kelvin in the $T_{e}$ can account for the differences we see.

The sulfur abundance reported in this paper agrees (although to a lesser extent than the neon) with those of Dopita \& Meatheringham (1991a, 1991b). The abundances are usually within a factor of 3 ; when the agreement is less good our abundances are always lower. Dopita \& Meatheringham (1991a, 1991b) have information on the $\mathrm{S}^{+}$and $\mathrm{S}^{+2}$ ions, and in several cases only upper limits for one or both ions could be derived. However, the contribution to the sulfur abundance in PNe comes mainly from the $\mathrm{S}^{+2}$ and $\mathrm{S}^{+3}$, and both these ions can be measured in the infrared. The abundances by Leisy \& Dennefeld (2006) are much higher than either presented in this paper or by Dopita \& Meatheringham (1991a, 1991b). The discrepancies are sometimes larger than a factor of 15 . Some of the sulfur abundances reported by Leisy \& Dennefeld (2006) are even higher than solar by factors of a few, which is difficult to interpret. This large difference cannot be accounted for by differences in the $\mathrm{S}^{+2}$ fractional abundance using infrared or optical lines which we have recalculated. The $[\mathrm{S} \mathrm{III]}$ line at $6312 \AA$ used by Leisy \& Dennefeld (2006) is blended with a He II line, which may add a fraction to the total sulfur abundance. In addition, for many cases Leisy \& Dennefeld (2006) could only measure upper limits to the $[\mathrm{S}$ III] line and they state that their sulfur abundance is very uncertain. It is possible that the ICFs used to derive the total sulfur abundance in Leisy \& Dennefeld (2006) may overestimate the contribution of $\mathrm{S}^{+3}$ by a large factor. Despite the uncertainties explained in our abundance determination we consider our sulfur abundances to be more accurate than the previous work as we have measured the important stages of ionization and complemented with the existing data in the literature.

SMP LMC 08 needs special mention. Leisy \& Dennefeld (2006) quote a much lower abundance of neon $\left(1.8 \times 10^{-5}\right)$ than the one we derive. We find that using their measured optical line at $3869 \AA$, extinction, $T_{e}$, and $N_{e}$ values, the $\mathrm{Ne}^{+2}$ fractional abundance is a factor 32 lower than using the infrared line. The infrared line at $15.5 \mu \mathrm{m}$ is very bright (Fig. 3 ) and could be measured easily. While we do not know the nature of this discrepancy, a small part of this discrepancy can be ascribed to difference in the extinction, since Leisy \& Dennefeld (2006) use a very low value $(C=0.01)$ compared to the one by Meatheringham \& Dopita (1991a) which we use, or uncertainties in the $T_{e}$. The IRS spectrum of the object shows that $\mathrm{Ne}^{+}$is also an important contributor to the total neon abundance (about $30 \%$ ) and this may be underestimated by Leisy $\&$ Dennefeld (2006). SMP LMC 08 also shows a large abundance of sulfur, but interestingly in this case Leisy \& Dennefeld (2006) 
quote a lower limit to its abundance which is a factor 10 higher than ours $\left(1.17 \times 10^{-5}\right)$ and of the order of the solar value $(1.4 \times$ $10^{-5}$ ). The $\mathrm{Ne} / \mathrm{S}$ abundance we find is about 9 , which is similar to the rest of the sample (but a bit on the lower end). This may imply that the $\mathrm{H} \beta$ flux we use for this object (Wood et al. 1987) to derive the elemental abundance is probably not adequate, and will render the abundance derived for this object uncertain.

\section{SUMMARY AND CONCLUSIONS}

We report the high-resolution Spitzer IRS observations of a sample of $18 \mathrm{PNe}$ in the LMC and seven in the SMC. The spectra cover the 10-37 $\mu \mathrm{m}$ wavelength range and show the usual fine-structure lines of neon, sulfur, and oxygen typically seen in PNe. Some nebulae also show high-excitation lines of argon and magnesium.

The abundances for neon and sulfur have been derived and compared to Galactic $\mathrm{PNe}$ and $\mathrm{H}$ II regions, $\mathrm{MC} \mathrm{H}$ in regions, and the solar values. The neon average abundances are $6.0 \times 10^{-5}$ and $2.7 \times 10^{-5}$ in the LMC and SMC, respectively. This is $\sim 1 / 3$ and $1 / 6$ of the average neon abundance of the Galactic PNe used for comparison. Given the uncertainties, these values agree well with the most quoted values for the LMC and SMC metallicity ( $1 / 3$ and $1 / 5$ solar, respectively).

The average $\mathrm{Ne} / \mathrm{S}$ ratio of the $\mathrm{MC} \mathrm{PNe}$ (23.5) is slightly higher than the average ratio for Galactic PNe (16), but the range of $\mathrm{Ne} / \mathrm{S}$ values is similar in both samples. These values are also similar to those found in $\mathrm{H}$ II regions. We believe that this is an indication that neon enrichment has either not occurred or remained modest in most of the nebulae. This agrees with the conclusion by Dopita et al. (1997) based on their derived abundances in a sample of LMC PNe. In fact, nucleosynthesis models suggest that this process occurs in a very narrow range of masses and thus statistically few objects would experience such enrichment. Four objects show a high Ne/S ratio. In two of them (SMP LMC 31 and SMP SMC 01) the high ratio is mainly due to the very low sulfur abundance of these objects, but together with SMP LMC 28 they show the highest neon abundance in the sample so there is a possibility that these $\mathrm{PNe}$ may have experienced some neon enrichment.

The range of neon abundances of $\mathrm{PNe}$ and $\mathrm{H}$ II regions is the same in the Milky Way, the LMC, and the SMC. The sulfur abundance of Galactic PNe and $\mathrm{H}$ II regions is also similar for both sets of objects. For the LMC it seems that some PNe show lower sulfur abundances than the $\mathrm{H}$ in regions, but the number of LMC H II regions to which we compare is not very large. The sulfur abundances of M33 H II regions have a similar range of abundances to those of the LMC and SMC PNe. Given the low number of objects in the sample of SMC PNe and $\mathrm{H}$ in regions we state that it seems that both types of objects show similar sulfur abundances but clearly more objects of both kinds are needed.

The two nebulae, SMP LMC 31 and SMP SMC 01, showing a clear lower sulfur abundance compared to the rest of the objects also show a $\mathrm{MgS}$ feature in their low-resolution IRS spectra. One could argue that some of the sulfur is depleted in dust. However, other objects which show the MgS feature do not have a lower sulfur abundance. This should be further investigated.

The PNe abundances derived are also compared to previous determinations by Leisy \& Dennefeld (2006) from optical line measurements and Dopita \& Meatheringham (1991a, 1991b) and Dopita et al. (1997) using photoionization models. The comparison shows that our derived neon abundance agrees very well with those by Dopita \& Meatheringham (1991a, 1991b) and to a lesser extent with those of Leisy \& Dennefeld (2006). The sulfur abundances we derive agree well for about half of the objects with the abundances determined by Dopita \& Meatheringham (1991a), 1991b) but for the rest of the PNe they are up to a factor of 3 lower. The sulfur abundances derived by Leisy \& Dennefeld (2006) are much higher than either the ones derived in this paper or the ones by Dopita \& Meatheringham (1991a, 1991b). The advantage of the abundances presented in this paper over previous work is that we have measured and used the most important stages of ionization and complemented existing data in the literature.

We thank an anonymous referee whose comments have improved the paper. J. B. S. would like to thank Duncan Farrah for reading parts of the manuscript. This work is based on observations made with the Spitzer Space Telescope, which is operated by the Jet Propulsion Laboratory, California Institute of Technology under NASA contract 1407. Support for this work was provided by NASA through contract number 1257184 issued by JPL/Caltech.

\section{REFERENCES}

Aller, L. H. 1983, ApJ, 273, 590

Aller, L. H., Keyes, C. D., Ross, J. E., \& O’Mara, B. J. 1981, MNRAS, 194, 613

Aller, L. H., et al. 1987, ApJ, 320, 159

Antia, M., \& Basu, S. 2005, ApJ, 621, L85

Asplund, M., Grevesse, N., \& Sauval, A. J. 2005, in ASP Conf. Ser. 336, Cosmic Abundances as Records of Stellar Evolution and Nucleosynthesis in Honor of David L. Lambert, ed. T. G. Barnes, III, \& F. N. Bash (San Francisco: ASP), 25

Bahcall, J. N., Basu, S., \& Serenelli, A. M. 2005, ApJ, 631, 1281

Bernard-Salas, J., Peeters, E., Sloan, G. C., Cami, J., Guiles, S., \& Houck, J. R. 2006, ApJ, 652, L29

Bernard-Salas, J., Pottasch, S. R., Beintema, D. A., \& Wesselius, P. R. 2001, A\&A, 367, 949

Bernard-Salas, J., et al. 2004, ApJS, 154, 271

Cohen, M., Megeath, T. G., Hammersley, P. L., Martin-Luis, F., \& Stauffer, J. 2003, AJ, 125, 2645

Corradi, R. L. M., et al. 2005, A\&A, 431, 555

de Graauw, T., et al. 1996, A\&A, 315, L345

Dopita, M. A., \& Meatheringham, S. J. 1991a, ApJ, 367, 115 1991b, ApJ, 377, 480

Dopita, M. A., et al. 1997, ApJ, 474, 188

Feldman, U., \& Widing, K. G. 2003, Space Sci. Rev., 107, 665

Fluks, M. A., et al. 1994, A\&AS, 105, 311
Grevesse, N., \& Noels, A. 1993, in Proc. Origin and Evolution of the Elements, ed. N. Prantzos, E. Vangioni-Flam, \& M. Cassé (Cambridge: Cambridge Univ. Press), 14

Grevesse, N., \& Sauval, A. J. 1998, Space Sci. Rev., 85, 161

Guiles, S., Bernard-Salas, J., Pottasch, S. R., \& Roellig, T. L. 2007, ApJ, 660, 1282

Henry, R. B. C., Kwitter, K. B., \& Balick, B. 2004, AJ, 127, 2284

Higdon, S. J. U., et al. 2004, PASP, 116, 975

Houck, J. R., et al. 2004, ApJS, 154, 18

Iben, I., \& Renzini, A. 1983, ARA\&A, 21, 271

Karakas, A. I., \& Lattanzio, J. C. 2003, Publ. Astron. Soc. Australia, 20, 393

Kim, S., et al. 1998, ApJ, 503, 674

Landi, E., Feldman, U., \& Doschek, G. A. 2007, ApJ, 659, 743

Lebouteiller, V., et al. 2007, ApJ, submitted

Leisy, P., \& Dennefeld, M. 2006, A\&A, 456, 451

Leisy, P., Dennefeld, M., Alard, C., \& Guibert, J. 1997, A\&AS, 121, 407

Magrini, L., Perinotto, M., Corradi, R. L. M., \& Mampaso, A. 2001, A\&A, 379, 90

Magrini, L., Vilchez, J. M., Mampaso, A., Corradi, R. L. M., \& Leisy, P. 2007, A\&A, 470, 865

Magrini, L., et al. 2003, A\&A, 400, 511

Marigo, P., Bernard-Salas, J., Pottasch, S. R., Tielens, A. G. G. M., \& Wesselius, P. R. 2003, A\&A, 409, 619

Martín-Hernández, N. L., et al. 2002, A\&A, 381, 606 
Meatheringham, S. J., \& Dopita, M. A. 1991a, ApJS, 75, 407 1991b, ApJS, 76, 1085

Meatheringham, S. J., Dopita, M. A., \& Morgan, D. H. 1988, ApJ, 329, 166 Meixner, M., et al. 2006, AJ, 132, 2268

Monk, D. J., Barlow, M. J., \& Clegg, R. E. S. 1988, MNRAS, 234, 583

Morgan, D. H., \& Parker, Q. A. 1998, MNRAS, 296, 921

Peña, M., Ruiz, M. T., \& Torres-Peimbert, S. 1997, A\&A, 324, 674

Pottasch, S. R., \& Bernard-Salas, J. 2006, A\&A, 457, 189

Pottasch, S. R., Bernard-Salas, J., \& Roellig, T. L. 2007, A\&A, 471, 865

Pottasch, S. R., \& Surendiranath, R. 2005, A\&A, 432, 139

Rubin, R. H., Simpson, J. P., Erickson, E. F., \& Haas, M. R. 1988, ApJ, 327, 377

Rubin, R. H., et al. 2007, MNRAS, 377, 1407

Sanduleak, N., MacConnel, D. J., \& Philip, A. G. D. 1978, PASP, 90, 621

Shaw, R. A., Stanghellini, L., Mutcher, M., Balick, B., \& Blades, J. C. 2001, ApJ, 548, 727

Shaw, R. A., Stanghellini, L., Villaver, E., \& Mutcher, M. 2006, ApJS, 167, 201
Stanghellini, L., Blades, J. C., Osmer, S. J., Barlow, M. J., \& Liu, X.-W. 1999, ApJ, 510, 687

Stanghellini, L., et al. 2002, ApJ, 575, 178

2003, ApJ, 596, 997

2005, ApJ, 622, 294

Surendiranath, R., Pottasch, S. R., \& García-Lario, P. 2004, A\&A, 421, 1051

Vermeij, R., \& van der Hulst, J. M. 2002, A\&A, 391, 1081

Villaver, E., Stanghellini, L., \& Shaw, R. 2003, ApJ, 597, 298 2004, ApJ, 614, 716

Wang, W., \& Liu, X.-W. 2007, MNRAS, in press

Werner, M., et al. 2004, ApJS, 154, 1

Wood, P. R., Meatheringham, S. J., Dopita, M. A., \& Morgan, D. H. 1987, ApJ, 320,178

Zijlstra, A. A., van Hoof, P. A. M., Chapman, J. M., \& Loup, C. 1994, A\&A, 290,228 